\title{
METAMEDIOS SOCIALES Y SERVICIO PÚBLICO: EL DEBATE EN TWITTER DE LOS PROGRAMAS FACTUALES DE BBC, RTVE Y CCMA
}

\section{Social metamedia and public service: The debate in Twitter for television programs on the BBC, RTVE, and CCMA}

\section{Pablo Gómez-Domínguez, Reinald Besalú-Casademont y Frederic Guerrero-Solé}
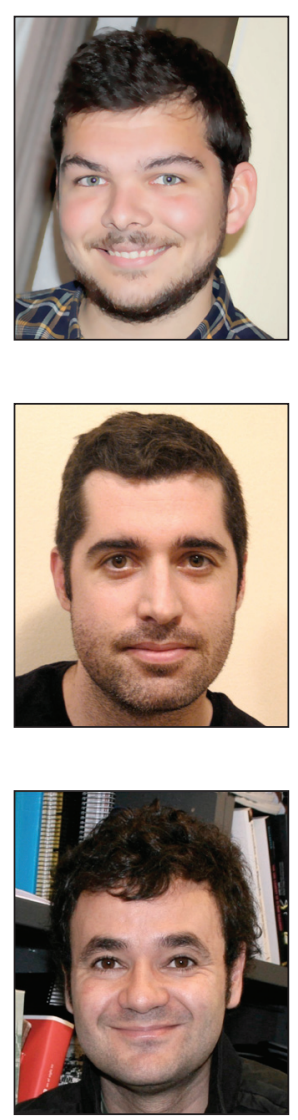

Pablo Gómez-Domínguez es graduado en comunicación audiovisual por la Universitat de Barcelona y doctorando del Departament de Comunicació de la Universitat Pompeu Fabra. Sus líneas de investigación se centran en los medios de servicio público, las audiencias digitales, el espacio catalán de comunicación y la calidad mediática. Es colaborador del Observatorio de la Producción Audiovisual de Catalunya, donde lleva a cabo análisis sobre televisión y redes sociales. http://orcid.org/0000-0002-0369-5616

pablo.gomez@upf.edu

Reinald Besalú-Casademont es licenciado en comunicación audiovisual y en sociología, y doctor en comunicación pública por la Universitat Pompeu Fabra (UPF). Es profesor visitante del Departamento de Comunicación de la UPF y miembro del grupo de investigación Unica. Sus intereses de investigación se centran en el estudio cuantitativo y cualitativo de las audiencias televisivas, así como en el análisis del espacio catalán de comunicación. Ha publicado en revistas científicas nacionales e internacionales.

http://orcid.org/0000-0002-2485-361X

reinald.besalu@upf.edu

Frederic Guerrero-Solé es licenciado en física y doctor en comunicación por la Universitat Pompeu Fabra, en cuyo Departamento de Comunicación ejerce como profesor lector. Sus principales líneas de investigación son las redes sociales, los efectos de los medios y la sociología de la comunicación. Ha publicado diversos artículos sobre Twitter en Information, communication and society y en Social science computer review.

http://orcid.org/0000-0001-8145-8707

frederic.guerrero@upf.edu

Universitat Pompeu Fabra, Departamento de Comunicación Roc Boronat, 138. 08018 Barcelona, España

\begin{abstract}
Las televisiones públicas tienen la misión de promover el debate en torno a temas de interés público. Twitter es el metamedio social escogido por los programas televisivos para cumplir esta tarea en la Red. Por ello, este estudio cuestiona la capacidad de las televisiones públicas para generar un debate efectivo, en usuarios y duración, a través de Twitter, así como la relación entre audiencia y audiencia social. Aplicamos una metodología cuantitativa que emplea una técnica de captura y tratamiento propia para examinar la actividad generada por los hashtags propuestos por 6 programas de televisión de $B B C$ One, TV3 y La 1 de TVE. Los resultados muestran que estos programas fallan en generar un debate público en Twitter. La discusión creada se concentra en un grupo recurrente de usuarios que canalizan el debate en torno a sus comentarios. Por último, podemos descartar la audiencia como factor predictivo de la audiencia social.
\end{abstract}

\section{Keywords}

Medios digitales; Metamedios; Televisión; Redes sociales; Audiencias; Audiencia social; Debate; Twitter; BBC; CCMA; TV3; RTVE; Hashtag. 


\section{Resumen}

Public television has a mission to promote debate around topics of public interest. Twitter is the social metamedia used by TV programs to fulfil this task online. This research analyzes the capability of public television to generate an effective debate through Twitter and also looks at the relationship between physical audience and social audience. We apply a quantitative methodology that employs our own capture and treatment technique to examine the activity generated by the hashtags proposed by six television programs on BBC One, TV3, and La 1. The main finding shows that these programs fail in their mission to generate a robust public debate in Twitter; instead the discussion is generated by a recurrent group of users that control the debate flow around their comments. Finally, the physical audience is not a predictive factor of the social audience.

\section{Palabras clave}

Digital media; Metamedia; Television; Social networks; Audiences; Social audience; Debate; Twitter; Hashtags; BBC; CCMA; TV3; RTVE.

Gómez-Domínguez, Pablo; Besalú-Casademont, Reinald; Guerrero-Solé, Frederic (2016). “Metamedios sociales y ser-

vicio público: el debate en Twitter de los programas factuales de BBC, RTVE y CCMA". El profesional de la información, v. 25, n. 3, pp. 384-394.

http://dx.doi.org/10.3145/epi.2016.may.08

\section{Introducción}

Los servicios públicos de radiodifusión europeos se encuentran en una fase de cambio y convergencia para adaptar su oferta a los nuevos medios o metamedios ${ }^{1}$ (Manovich, 2008). Tradicionalmente esas corporaciones han portado el estandarte de la innovación en todas las fases de creación y difusión audiovisual (Harrison; Wessels, 2005). Actualmente, la escasa financiación de los departamentos digitales, las lagunas legales en la regulación del sector de los metamedios (Brevini, 2010), la falta de asimilación de los cambios en los hábitos de consumo y el escaso reciclaje de las rutinas profesionales dificulta que los servicios públicos de radiodifusión aprovechen las oportunidades del entorno digital (Campos-Freire, 2015).

En el ecosistema de medios digitales, los metamedios sociales han supuesto un cambio en el modelo clásico de comunicación de masas. Al one-to-many de la radiodifusión se añade el one-to-one, many-to-one y many-to-many del servicio público multimedia (SPM) (Chadwick, 2006). Los metamedios sociales "traen consigo una nueva oportunidad para el diálogo entre el contenido y las audiencias" (GarcíaDe-Torres et al., 2011, p. 612). Así, los SPM consideran entre sus misiones la estructuración de canales para comunicarse con sus usuarios-audiencia y fomentar entre ellos una esfera pública de debate mediático (Larsen, 2010).

Twitter se ha convertido en uno de los canales de debate más relevantes entre los programas de las corporaciones públicas y sus usuarios (Buschow; Schneider; Ueberheide, 2014). Mediante la propuesta de hashtags relacionados con la marca del programa o el tema, los SPM promueven la participación de su audiencia. Aunque la intención de las televisiones públicas con el hashtag es generar interacción (engagement), existen múltiples factores que pueden afectar la creación del debate online. Entre ellos, las barreras demográficas que inciden sobre los hábitos de consumo, elementos como el horario de emisión o la duración del programa, así como la actualidad mediática, que puede desviar la atención del usuario hacia otros canales o contenidos (GalindoRubio; Fernández-Blanco; Alameda-García, 2014).
Este estudio plantea si los medios públicos son capaces de generar un debate efectivo y duradero en Twitter. Efectivo en términos del número de usuarios involucrados en él y de las características de su participación. Duradero, si el programa es capaz de mantener este debate en el tiempo. En definitiva, nos proponemos analizar cómo se organiza el debate digital, a través de un metamedio social, en torno al contenido de programas de servicio público de género factual.

Twitter se ha convertido en uno de los canales de debate más relevantes entre los programas de las corporaciones públicas y sus usuarios

\section{Marco teórico}

Las corporaciones públicas deben cumplir una serie de obligaciones y misiones que justifiquen su presencia en un entorno audiovisual predominantemente privado y digital (Enli, 2008). Debrett (2009, p. 809) considera que la convergencia empuja a los prestadores públicos a "proveer contenidos de calidad que abarquen la predisposición a innovar sin dejarse llevar por la audiencia esperada". A la calidad se liga la programación de formatos informativos, considerados por autores como Curran et al. (2009) fundamentales para la construcción y el mantenimiento de las sociedades democráticas. Se trata de espacios clave que ofrecen a la audiencia información necesaria para que participe en la vida pública (Thomass, 2010). Como consecuencia, formatos de tipo factual como el debate político o el documental ocupan una parte inamovible en las parrillas de las televisiones públicas europeas (Walzer; Retis, 2008).

La digitalización también ha transformado los hábitos de consumo de la audiencia. Steemers (2002) preveía un cambio en la relación entre medio y público que pasaba por una oferta más personalizada y la creación de medios de comunicación bilateral. Más tarde, Bruns (2008) afirma que estos cambios han provocado una fragmentación en la audiencia 
de masas. Este fenómeno se suma a un incremento de los canales de comunicación en forma de webs, aplicaciones móviles y redes sociales. García-Avilés (2012) concluye que estos cambios han generado una suerte de audiencia social caracterizada por la multiplicidad de perfiles y la búsqueda constante de participación. En suma, la audiencia ha empujado a los servicios públicos a añadir la interactividad como nuevo pilar de su oferta (Jakubowicz, 2010).

Este nuevo contexto mediático cuestiona la capacidad de los servicios públicos multimedia (SPM) para generar una esfera pública de debate (Habermas, 1989). Para Dahlgren (2009), esta esfera tiene que actuar como mediadora entre las instituciones, los actores públicos y la sociedad, poniéndolos en contacto a través de una plataforma común como los medios de comunicación. Como sostienen Gripsrud (2007) o Azurmendi et al. (2015), los SPM tienen que favorecer un doble flujo de comunicación y ofrecer canales que faciliten el contacto entre los diferentes actores de la esfera de debate. En este punto, los metamedios sociales muestran idoneidad como plataformas para que, a través de sus contenidos, las corporaciones públicas generen un debate público, abierto y duradero (Van-Dijk; Poell, 2015).

\section{La audiencia ha empujado a los servicios públicos a añadir la interactividad como nuevo pilar de su oferta}

La red más popular escogida por los programas de televisión para generar debate es Twitter. La inmediatez, la conversación pública o la extensión de los mensajes la demuestra apta para esta finalidad (Giglietto; Selva, 2014). En España y Gran Bretaña Quintas-Froufe, González-Neira y DíazGonzález (2015) y Anstead y O'Loughlin (2011) sostienen que Twitter, como parte del fenómeno de las segundas pantallas, es el servicio más utilizado para comentar programas durante la emisión. En el caso de los medios públicos, Bonini y Sellas (2014) afirman que el potencial de esta red radica en la planificación de estrategias de participación por parte de los programas, y añaden que Twitter facilita a los SPM establecer un diálogo real y paralelo con el público.

Finalmente, Buschow, Schneider y Ueberheide (2014) comprueban que son el género factual y los formatos de deba- te y documental los que atraen mayor actividad en Twitter. Para estos autores los sucesos de actualidad, el tema tratado y los conductores del programa son indicadores fundamentales para organizar un debate en red. En el caso de los programas de debate político, son numerosos los estudios sobre la incidencia de Twitter y la información que puede extraerse de esta red en cuanto a las características de los usuarios, sus tendencias ideológicas (Guerrero-Solé; Corominas-Murtra; López-González, 2014) o la polarización del debate (Guerrero-Solé, 2015). En estos estudios el hashtag ha sido el parámetro seleccionado para determinar la inclusión de un mensaje concreto en una conversación. El hashtag es uno de los principales recursos empleados por las televisiones para ofrecer a su audiencia una referencia de inclusión a su contenido en una conversación paralela a la emisión (Greer; Ferguson, 2011).

\section{Preguntas de investigación}

Considerando esta revisión teórica, formulamos dos preguntas de investigación para desvelar cómo se organiza el debate público a través del metamedio social Twitter en los programas de género factual, evaluando su capacidad para promover una discusión efectiva en número de participantes y regular en el tiempo. También cuestionamos si existe una relación proporcional entre el número de espectadores del programa y el de usuarios de Twitter como factor explicativo de la generación de debate.

P1: A través de sus programas de género factual, ¿'Las televisiones públicas son capaces de generar un debate público en Twitter?

P1.1: ¿Cómo se distribuye el debate en Twitter antes, durante y después de la emisión de los programas?

P1.2: ¿Qué tipo de usuarios participan activamente en la red social durante las ediciones de cada programa?

P1.3: ¿Existen usuarios que actúan como líderes de opinión (influencers) en los debates analizados?

P2: ¿Qué papel juega la audiencia como factor predictivo del número de usuarios que participan en el debate?

P2.1: ¿̇nfluye la edad de la audiencia de los programas analizados en las características del debate público generado en Twitter?

\begin{tabular}{|c|c|c|c|c|c|c|}
\hline Corporación & Canal & Share $^{3}$ & Programa & Formato & Franja & Twitter \\
\hline & \multirow{2}{*}{$\begin{array}{l}B B C \\
\text { One }\end{array}$} & \multirow{2}{*}{$23 \%$} & BBC Panorama & Documental & \multirow{2}{*}{ Late peak time $e^{2}$} & @BBCPanorama \\
\hline & & & $B B C$ Question time & Debate & & @bbcquestiontime \\
\hline & \multirow{2}{*}{ La 1} & \multirow{2}{*}{$17 \%$} & Comando actualidad & Documental & Prime-time & @comandotve \\
\hline & & & El debate de La 1 & Debate & Latenight & @debatedela1_tve \\
\hline \multirow[b]{2}{*}{$\begin{array}{l}\text { Corporació Catalana } \\
\text { Mitjans Audiovisuals,SA }\end{array}$} & \multirow[b]{2}{*}{ TV3 } & \multirow[b]{2}{*}{$13 \%$} & 30 minuts & Documental & \multirow[b]{2}{*}{ Prime-time } & @30minuts \\
\hline & & & cat & Debate & & @puntcatTV3 \\
\hline
\end{tabular}




\section{Metodología}

\subsection{Corpus. Corporación, canales y programas}

El corpus está formado por una muestra estratificada en tres niveles: corporación pública, canal de televisión y programa.

En el primer nivel se seleccionaron tres corporaciones públicas con criterios geográficos -ámbito internacional, nacional y autonómico-, y de sistema mediático -liberal y pluralista polarizado (Hallin; Mancini, 2004)-.

En el segundo nivel se empleó un criterio de audiencia y antigüedad para escoger los canales de televisión.

Por último, se aplicaron criterios de género televisivo, de formato y de presencia en Twitter para escoger los programas. Estos datos se han sintetizado en la tabla 1.

Los programas seleccionados fueron:

- BBC Panorama (BBCpan): documental televisivo sobre temas que varían según la actualidad mediática.

- $B B C$ Question time ( $B B C q t)$ : debate itinerante que recorre Reino Unido reuniendo a representantes políticos y ciudadanos sin una temática recurrente.

- Comando actualidad (caTVE): reportaje documental que se centra en temas sociales o de sucesos.

- El Debate de La 1 (dbtTVE): debate con un tema central por edición que reúne a expertos y representantes políticos en una discusión en directo.
- 30 minuts (30minutsTV3): comparte formato y duración con BBCpan. Sus temas giran en torno a la política y la economía.

- Punt Cat (.catTV3): debate fundamentalmente político.

El núcleo de discusión está compuesto por un grupo específico y reducido de usuarios

\subsection{Aplicación. Captura y tratamiento de la muestra}

Seleccionamos un período de 35 días continuos, escogidos de forma aleatoria, desde el 26 de octubre ${ }^{4}$ al 30 de noviembre de 2015. Esta muestra puede considerarse suficiente para evaluar la efectividad del debate, su duración y el cambio de temática de los programas. Durante este período se llevó a cabo un seguimiento de las cuentas oficiales de Twitter de los programas analizados que permitió comprobar el hashtag utilizado por cada uno de ellos para generar debate alrededor de sus ediciones. La tabla 2 recopila estos hashtags, así como su relación con otras variables.

Los hashtags capturados revelan información sobre la creación de debate de cada programa. Por ello hemos añadido otras variables como la sobreimpresión de la etiqueta en pantalla y la sobreimpresión de los tuits en directo. Este recurso establece un "puente" entre los espectadores y el

Tabla 2. Distribución de hashtags en función del programa, la semana de emisión, la sobreimpresión del hashtag (\#) y la sobreimpresión de los tuits en directo (TW)

\begin{tabular}{|c|c|c|c|c|}
\hline Programa & Hashtag & Semana & Sobreimpresión \# & Sobreimpresión TW \\
\hline \multirow{5}{*}{ BBC Panorama } & \#MentalHealthCrisis & $26 / 10 / 2015$ & & \\
\hline & \#painkillers & $2 / 11 / 2015$ & & \\
\hline & \#bbcpanorama & $9 / 11 / 2015$ & & \\
\hline & \#BBCPanorama & $16 / 11 / 2015$ & & \\
\hline & \#BBCPanorama & $23 / 11 / 2015$ & & \\
\hline BBC Question time & $\# b b c q t$ & Siempre & & \\
\hline \multirow{5}{*}{ Comando actualidad } & \#Comandoactualidad & $26 / 10 / 2015$ & & \\
\hline & \#ComandoActualidad/\#EnganchadosRed & $2 / 11 / 2015$ & & \\
\hline & \#comandoactualidad/\#Derrumbados & $9 / 11 / 2015$ & & \\
\hline & \#lonuncavisto & $16 / 11 / 2015$ & & \\
\hline & \#comandoactualidad/\#jamonjamon & $23 / 11 / 2015$ & & \\
\hline El debate de la 1 & \#elDBT & Siempre & & \\
\hline \multirow{5}{*}{30 minuts } & -- & -- & & \\
\hline & \#PensionsTV3 & $2 / 11 / 2015$ & & \\
\hline & \#JihadTV3 & $9 / 11 / 2015$ & & \\
\hline & \#FugirEITV3 & $16 / 11 / 2015$ & & \\
\hline & \#PreuPorcTV3 & $23 / 11 / 2015$ & & \\
\hline \multirow{5}{*}{ cat } & \#ForcadellTV3 & $26 / 10 / 2015$ & \multirow{5}{*}{$V$} & \\
\hline & \#puntcatTV3 & $2 / 11 / 2015$ & & \\
\hline & \#BañosTV3/\#AlbiolTV3 & $9 / 11 / 2015$ & & \\
\hline & \#EstatlslàmicTV3 & $16 / 11 / 2015$ & & \\
\hline & \#MuntéTV3/\#EmpresarisTV3 & $23 / 11 / 2015$ & & \\
\hline
\end{tabular}


metamedio y dota de oficialidad al hashtag propuesto por el programa en la red social. Así mismo recopilamos la información de la audiencia de cada programa en relación con el número total de espectadores y grupos de edad. Durante los 35 días del período especificado se almacenaron todos los mensajes de Twitter que contenían alguno de los hashtags propuestos por los programas de la tabla 1. Para esta captura se utilizó una aplicación que utiliza la API Search de Twitter y que proporciona, entre otros datos, la hora y fecha de publicación, el autor y el cuerpo del mensaje. De todos los mensajes recogidos se seleccionaron únicamente aquellos publicados entre las 4 horas anteriores a la emisión del programa (pre-communication), durante su emisión (parallel communication) y 24 horas después de ésta (followup-communication), período temporal durante el que se recogen la mayor parte de mensajes que contienen un determinado hashtag televisivo (Anstead; O'Loughlin, 2011).

\section{Resultados}

\subsection{Debate público en Twitter}

El primer análisis que presentamos se refiere al total de usuarios activos durante la emisión de cada programa, así como al número total de comentarios publicados. Este dato nos informa del volumen real de participantes en el debate online. Se estableció un mínimo de usuarios activos para incluir los programas en la comparativa y excluir aquellos que por volumen no generan suficiente actividad. Marcamos la cifra de $500^{5}$ usuarios como mínimo para incluir un programa en esta comparación. Consideramos, dados los resultados, que se trata de una cifra razonable en términos de participantes de una discusión en red.

La tabla 3 muestra que sólo BBCqt supera, por un amplio margen, el límite de los 500 usuarios activos durante la emisión de sus cinco programas. Por otro lado, únicamente las ediciones de .catTV3 con los hashtags \#ForcadellTV3, \#BañosTV3/\#AlbiolTV3 y las de 30minutsTV3 con la etiqueta
Tabla 3. Distribución de usuarios únicos en Twitter (UTW) y comentarios publicados (TW) en función del programa, la fecha de emisión y el hashtag utilizado por una cifra mayor o igual a 500 usuarios ( $\#>=500$ )

\begin{tabular}{|c|c|c|c|c|}
\hline Programa & Emisión & $\#>=500$ & uTW & TW \\
\hline \multirow{4}{*}{ catTV3 } & 29/10/2015 & \#ForcadellTV3 & 1.488 & 3.820 \\
\hline & $5 / 11 / 2015$ & \#puntcatTV3 & 929 & 1.695 \\
\hline & $12 / 11 / 2015$ & $\begin{array}{l}\text { \#BañosTV3 } \\
\text { \#AlbiolTV3 }\end{array}$ & 3.904 & 9.166 \\
\hline & $19 / 11 / 2015$ & \#Estat/slàmicTV3 & 696 & 1.232 \\
\hline \multirow{2}{*}{ 30mintusTV3 } & $22 / 11 / 2015$ & \#FugirEITV3 & 1.144 & 2.087 \\
\hline & $8 / 11 / 2015$ & \#PensionsTV3 & 498 & 732 \\
\hline dbtTVE & $25 / 11 / 2015$ & \#elDBT & 823 & 4.618 \\
\hline \multirow{5}{*}{$B B C Q T$} & 29/10/2015 & \multirow{5}{*}{ \#bbcqt } & 11.814 & 42.546 \\
\hline & $5 / 11 / 2015$ & & 15.578 & 43.279 \\
\hline & $12 / 11 / 2015$ & & 14.158 & 46.134 \\
\hline & $19 / 11 / 2015$ & & 21.008 & 60.219 \\
\hline & $26 / 11 / 2015$ & & 14.708 & 47.612 \\
\hline
\end{tabular}

\#FugirEITV3 duplican o triplican la cifra mínima establecida. El caso de dbtTVE es aún más llamativo ya que, aunque comparte formato y franja de emisión con .catTV3 y BBCqt, sólo supera los 500 usuarios en una de sus ediciones. Los programas caTVE y BBCpan no han superado en ninguno de sus programas el margen establecido.

La relación entre hashtag y tuits revela que BBCqt mantiene estable el número de comentarios de los usuarios que etiquetan \#bbcqt. Esta tendencia alcanza su máximo en la emisión del 19 de noviembre, cuando el programa británico llega a los 60.219 comentarios. El resto de los programas presenta cifras irregulares con máximos en algunas de sus ediciones. Por ejemplo .catTV3 y 30minutsTV3 en los días 12 y 22 de noviembre respectivamente. Esta situación puede apuntar a su temática: independencia de Catalunya y Estado Islámico en cada caso, como causantes del incremento de comentarios.

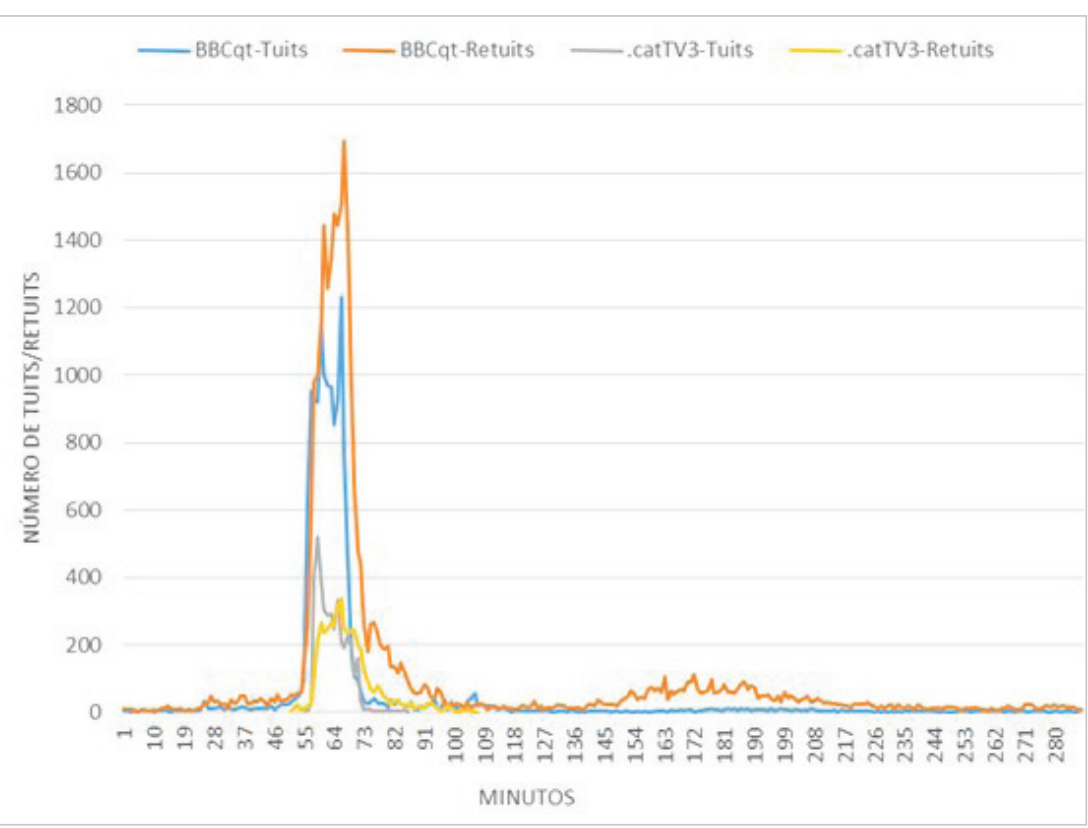

Gráfico 1. Distribución de la actividad de los usuarios durante el programa (minutos) en función del número de tuits y retuits publicados
Examinamos la actividad de los usuarios antes, durante y después de la emisión. Los resultados confirman que la distribución se replica en todos los casos estudiados: una actividad mínima antes de la emisión que se incrementa con la propuesta del hashtag (4h antes). El número de tuits y retuits alcanza su máximo en el transcurso del programa y el flujo de actividad decae a medida que éste acaba. Los datos revelan que tras la finalización del programa (24h después) la discusión queda prácticamente silenciada. A fin de mostrar esta tendencia, el gráfico 1 recoge los resultados de los dos programas con mayor número de tuits y retuits en cada fase. Esta selección se realiza a efectos de visualización, ya que todos los programas presentan el mismo flujo de actividad.

También se han seleccionado los casos 
Tabla 4. Distribución de usuarios, tuits publicados (TWp), tuits publicados por usuario y programa (TWu/p), retuits publicados (RTp), retuits por usuario y programa (RTu/p) y retuits recibidos (RTr) y media de seguidores en función del número de programas en los que participan los usuarios de $B B C q t$ y .catTV3

\begin{tabular}{|c|c|c|c|c|c|c|c|c|c|}
\hline \multicolumn{10}{|c|}{ BBCqt } \\
\hline $\begin{array}{l}\text { Programas en los } \\
\text { que participan }\end{array}$ & Usuarios & TWp & TWu/p & RTp & RTu/p & TW/RT & $\mathrm{RTr}$ & $\begin{array}{c}\text { RTr por usuario } \\
\text { y programa }\end{array}$ & $\begin{array}{l}\text { Promedio de seguido- } \\
\text { res por usuario }\end{array}$ \\
\hline 5 & 976 & 18.606 & 3,81 & 28.956 & 5,93 & 0,64 & 55.320 & 11,34 & 2.494 \\
\hline 4 & 1.704 & 14.599 & 2,14 & 25.586 & 3,75 & 0,57 & 28.691 & 4,21 & 1.957 \\
\hline 3 & 3.213 & 14.817 & 1,54 & 24.848 & 2,58 & 0,60 & 25.999 & 2,70 & 1.607 \\
\hline 2 & 7.579 & 14.285 & 0,94 & 27.233 & 1,80 & 0,52 & 19.500 & 1,29 & 3.345 \\
\hline 1 & 40.773 & 23.001 & 0,56 & 47.859 & 1,17 & 0,48 & 23.956 & 0,59 & 1.381 \\
\hline \multicolumn{10}{|c|}{.catTV3 } \\
\hline 4 & 30 & 295 & 2,46 & 493 & 4,11 & 0,60 & 1.539 & 12,83 & 20.278 \\
\hline 3 & 144 & 508 & 1,18 & 1.007 & 2,33 & 0,50 & 1.382 & 3,20 & 1.963 \\
\hline 2 & 692 & 1.388 & 1,00 & 2.396 & 1,73 & 0,58 & 3.045 & 2,20 & 1.765 \\
\hline 1 & 5.081 & 3.890 & 0,77 & 5.936 & 1,17 & 0,66 & 3.852 & 0,76 & 817 \\
\hline
\end{tabular}

de BBCqt y de .catTV3 para mostrar la dimensión real del debate en cuanto a usuarios participantes. Hemos sintetizado en la tabla 4 el número de usuarios que se mantienen activos durante todas las ediciones. Otros datos de interés añadido son las características de esta audiencia social en cuanto a interacción y perfil (tuits, retuits, seguidores, etc.). Los resultados de los usuarios que participan en los 5 y 4 programas de $B B C$ y $T V 3$ respectivamente, podrían estar sesgados por el hecho de que entre ellos se encuentran las cuentas de BBCqt y .catTV3. Sin embargo, si prescindimos de este usuario, sobre todo en el caso de los RTr (14.811 y 1.117), las proporciones continúan siendo similares (en lugar de una proporción de 11,34 y 12,83 retuits por usuario y programa obtenemos 8,30 y 3,57 , muy superior en $B B C q t$ y superior en .catTV3 a la del resto de los grupos de usuarios). Los que más participan en las conversaciones con la etiqueta \#bbcqt y las de .catTV3 son también los más activos, los que redifunden más información y los que son más redifundidos. Además son los que publican más contenidos originales, a diferencia de los que sólo participan en uno o dos programas, que tienden mucho más a redifundir contenidos que los demás.

Sin embargo el número de seguidores no sigue la misma tendencia que la actividad y el retuit. En un análisis más detallado se observa que entre los usuarios más seguidos hay una proporción mayor de los que sólo participan en dos debates. Sin embargo este mismo hecho no cambia las tendencias en el resto de variables observadas.

Los datos indican que el núcleo de discusión está compuesto por un grupo específico y reducido de usuarios. Estos actúan como líderes de opinión (influencers), canalizadores del debate propuesto por el programa mediante el hashtag. En la tabla 5 se muestran las cuentas de Twitter más influyentes en la conversación generada en torno a los programas de BBCqt y .catTV3 en función de la edición.

Tabla 5. Usuarios de Twitter más influyentes en los debates de BBCqt y .catTV3

\begin{tabular}{|c|c|c|c|c|c|}
\hline \multicolumn{6}{|c|}{ Influencers (@) } \\
\hline & $26 / 10 / 15$ & $2 / 11 / 15$ & $9 / 11 / 15$ & $16 / 11 / 15$ & $23 / 11 / 15$ \\
\hline \multirow{6}{*}{$B B C q t$} & bbcquestiontime & bbcquestiontime & bbcquestiontime & bbcquestiontime & bbcquestiontime \\
\hline & Bbcnews & ChukaUmunna & ParisLees & mehdirhasan & bbcnews \\
\hline & kdugdalemsp & piersmorgan & StigAbell & piersmorgan & mattforde \\
\hline & Afneil & jeremycorbyn & sajidjavid & frankieboyle & krishgm \\
\hline & TheSNP & GeorgeAylett & UKIP & andyburnhammp & MattHancockMP \\
\hline & AngrySalmond & TheGreenParty & HuffPostUK & caitlinmoran & Georgegalloway \\
\hline \multirow{9}{*}{ catTV3 } & ForcadellCarme & tv3cat & cupnacional & tv3cat & \\
\hline & tv3cat & ConvergenciaCAT & tv3cat & $324 c a t$ & \\
\hline & JuntsPelSi & JuntsPelSi & $324 c a t$ & puntcatTV3 & \\
\hline & Esquerra_ERC & puntcatTV3 & toniaira & orioljara & \\
\hline & $324 c a t$ & $324 c a t$ & puntCatTV3 & Nitsuga000 & \\
\hline & puntcatTV3 & sergipicazo & PPCatalunya & USConsulateBCN & \\
\hline & cupnacional & MIQUELLARA & poloniatv3 & & \\
\hline & ConvergenciaCAT & poloniatv3 & & & \\
\hline & & La_Directa & & & \\
\hline
\end{tabular}


El caso de $B B C q$ t revela que es la cuenta oficial del programa la que lidera el debate durante todas las ediciones analizadas. El resto de los usuarios, la mayoría de ellos partidos políticos (theSNP o UKIP) y líderes (kdugdalemsp o jeremycorbyn), varían según el programa. Podemos afirmar que existe un claro componente temático en la configuración final de los líderes de opinión.

Por otra parte .catTV3 replica en cierta medida esta situación, aunque la cuenta oficial del programa no tiene el mismo peso que en el caso de BBCqt. Entre el resto de usuarios abundan los partidos políticos (Esquerra_ERC o cupnacional) así como un líder (ForcadellCarme). Ambos comparten entre sus influencers otros programas o canales (bbcnews o 324cat) y algún presentador de la cadena (piersmorgan) (tabla 5).

Algunos factores que pueden explicar los niveles de audiencia social de los programas pasan por la estrategia que utilizan en Twitter

\subsection{Audiencia social}

Este análisis requiere:

- datos de audiencia -total de espectadores- de cada programa, extraídos de Kantar Media en el caso de España;

- datos de audiencia social -número de usuarios de Twitter- contabilizados mediante una aplicación propia.

Dada la necesidad de estos datos, procuramos obtener las cifras de la audiencia británica a través de tres vías:

- la Broadcasters Audience Research Board (BARB), homólogo de Kantar Media en Reino Unido;

- la $B B C$;

- la sección especializada en medios del diario The guardian.

A pesar de nuestros esfuerzos, la obtención de estos datos no ha sido posible. Esto se debe a que la BARB fija un elevado precio por ellos, la $B B C$ acuerda con ésta la confidencia- lidad de los datos y The guardian sólo dispone de cifras de espectadores para los programas más vistos, entre los que no se encuentran BBCqt y BBCpan. Por esto, al menos en este apartado, realizamos la comparativa de los programas emitidos en España.

Con las especificaciones del apartado anterior sobre el mínimo de usuarios considerados para la comparativa ( $>=500)$, la tabla 6 muestra la relación entre espectadores y usuarios en función del programa y el hashtag, así como el segmento demográfico predominante.

\section{El número de tuits y retuits alcanza su} máximo en el transcurso del programa y el flujo de actividad decae a medida que éste acaba. Tras la finalización del programa (24h después) la discusión queda prácticamente silenciada

Con estos resultados y atendiendo a los datos proporcionados por Kantar Media, observamos que tanto los programas de La 1 (caTVE y dbtTVE) como los de TV3 (30minutsTV3 y .catTV3) tienen perfiles de público muy envejecidos. En todos ellos el porcentaje de espectadores de más de 45 años es superior a la media para el total de la televisión (en todos los casos, más del $60 \%$ de los espectadores se sitúan en estas franjas de edad). También la presencia de espectadores de más de 65 años es más alta de lo habitual. Esto podría explicar en parte la baja audiencia social, considerando el hecho de que el uso de redes sociales como Twitter tiene un claro sesgo de edad.

La emisión de 30minutsTV3 con más usuarios y tuits (\#fugirEITV3) es aquella en la que el porcentaje de espectadores menores de 45 años fue mayor (35\%). Lo mismo sucede con .catTV3, cuya emisión con más audiencia social (\#BañosTV3 y \#A/biolTV3) obtuvo un porcentaje de espectadores menores de 45 años inusualmente alto (35,9\%); y además por encima de la media para el total de la televisión (que fue del $34,6 \%)$.

Tabla 6. Relación entre espectadores y usuarios de Twitter en función del programa y el grupo demográfico (franja de edad). Elaborado a partir de datos de Kantar Media y propios

\begin{tabular}{|c|c|c|c|c|c|}
\hline Fecha & Programa & Hashtag & Espectadores & Usuarios & Grupo demográfico \\
\hline 29/10/15 & \multirow{4}{*}{ catTV3 } & \#ForcadellTV3 & 269.000 & 1.488 & \multirow{7}{*}{$45-64$} \\
\hline $5 / 11 / 15$ & & \#puntcatTV3 & 224.000 & 929 & \\
\hline $12 / 11 / 15$ & & \#BañosTV3/\#AlbiolTV3 & 288.000 & 3.904 & \\
\hline $19 / 11 / 15$ & & \#EstatlslàmicTV3 & 190.000 & 696 & \\
\hline $22 / 11 / 15$ & \multirow{2}{*}{ 30mintusTV3 } & \#FugirEITV3 & 631.000 & 1.144 & \\
\hline $8 / 11 / 15$ & & \#PensionsTV3 & 520.000 & 498 & \\
\hline $25 / 11 / 15$ & dbtTVE & \#eIDBT & 309.000 & 823 & \\
\hline $29 / 10 / 15$ & \multirow{5}{*}{$B B C q t$} & \multirow{5}{*}{$\# b b c q t$} & & 11.814 & \multirow{5}{*}{2} \\
\hline $5 / 11 / 15$ & & & & 15.578 & \\
\hline $12 / 11 / 15$ & & & & 14.158 & \\
\hline $19 / 11 / 15$ & & & & 21.008 & \\
\hline $26 / 11 / 15$ & & & & 14.708 & \\
\hline
\end{tabular}


El número de usuarios y tuits de los programas varía también en función de su audiencia total. Parece lógico pensar que cuantos más espectadores alcanzan estos programas, más probabilidades hay de que algunos de ellos interactúen en Twitter a partir de las etiquetas propuestas, como de hecho así sucede. Las emisiones de .catTV3 y 30minutsTV3 mencionadas son las que obtuvieron mayor audiencia de entre todas las analizadas (288.000 y 631.000 espectadores respectivamente). Sin embargo, ha resultado evidente que la audiencia por sí misma no predice la audiencia social, ya que .catTV3 por ejemplo, obtiene unas audiencias claramente menores a las de 30minutsTV3, y en cambio su audiencia social es en términos generales mayor. En este sentido, apuntamos como posible factor explicativo la sobreimpresión de los tuits de la audiencia en la emisión televisiva, hecho que no se produce en 30minutsTV3 y sí en .catTV3, lo que podría suponer un mayor incentivo para la interacción en Twitter alrededor de los temas tratados en el programa.

\section{Las emisiones de 30minutsTV3 y .catTV3 con más tuits son aquellas en las que el porcentaje de espectadores menores de 45 años fue mayor}

La tabla 7 recoge el número total de usuarios de Twitter existentes por cada 1.000 espectadores. Esto prueba que no se puede establecer por ahora una relación proporcional entre la audiencia y la audiencia social. Se descarta que los datos de audiencia puedan ser actualmente predictores validos de la audiencia en red de un programa.

\section{Conclusión y discusión}

Los generalmente pobres resultados de audiencia social de los programas de género factual estudiados ponen en duda parte de las funciones de servicio público de los operadores que los emiten, entre cuyas obligaciones se incluye la de promover el debate público en torno a temas de interés social. A pesar de que los nuevos medios ofrecen a los servicios públicos multimedia la oportunidad de conectar más efectivamente con su audiencia, nuestros datos indican que no cumplen con el objetivo de crear una conversación con ella en forma de debate.
El programa que genera un mejor debate en términos de participación y duración, muestra que no existe un gran número de usuarios que contribuyen significativamente a la discusión. De hecho, los que participan de forma esporádica no pueden ser considerados parte de un debate consolidado por el programa a través de sus emisiones regulares. Estos usuarios ocasionales (lurkers) (Fazeen; Dantu; Guturu, 2011) llevan a concluir erróneamente a los programas que sus contenidos generan una discusión altamente participativa. Prueba de este error es que, en un análisis de su audiencia social, la dirección de redes del programa BBCqt afirma que su estrategia para generar un debate regular online es un éxito ${ }^{6}$.

\section{No se puede establecer por ahora una relación proporcional entre la audiencia y la audiencia social}

Algunos factores que pueden explicar los niveles de audiencia social de los programas, pasan precisamente por la estrategia que utilizan en relación con Twitter. Hay indicios de que el hecho de sobreimpresionar los hashtags y/o los tuits durante la emisión puede repercutir en una mayor actividad en la red. En ocasiones este factor puede resultar incluso más decisivo que el número de espectadores de un programa, como sucede en los casos de .catTV3 y 30minutsTV3.

Otro factor a tener en cuenta es el formato del programa. Los resultados apuntan que los documentales analizados, a excepción de 30minutsTV3, no generan ningún debate significativo en Twitter. Parece plausible afirmar que los debates televisivos tendrían más facilidad para trasvasar su discusión al metamedio. Consecuentemente el tema tratado no parece influir en los programas de este formato, cosa que sí sucede en el documental, donde 30minutsTV3 obtiene sus mejores resultados con dos ediciones dedicadas al Estado Islámico.

La cultura mediática también tiene un papel clave en la participación de la audiencia en debates online. Así lo confirman nuestros datos y autores como Anstead y O'Loughlin (2011) o Berrocal-Gonzalo et al. (2014). Los primeros consideran que es común en el caso del público británico atender con especial atención a los formatos de debate político como

Tabla 7. Número de usuarios de Twitter que corresponden por cada 1.000 espectadores en función del hashtag y el programa. Elaborado a partir de datos de Kantar Media y propios

\begin{tabular}{|c|c|c|c|}
\hline Programa & Hashtag & Espectadores & $\begin{array}{c}\text { Usuarios de TW por cada } 1.000 \\
\text { espectadores }\end{array}$ \\
\hline \multirow{4}{*}{.$c a t T V 3$} & \#ForcadellTV3 & 269.000 & 6 \\
\hline & \#puntcatTV3 & 224.000 & 4 \\
\hline & \#BañosTV3/\#AlbiolTV3 & 288.000 & 14 \\
\hline & \#EstatlslàmicTV3 & 190.000 & 4 \\
\hline \multirow{2}{*}{ 30minutsTV3 } & \#FugirEITV3 & 631.000 & 2 \\
\hline & \#PensionsTV3 & 520.000 & 1 \\
\hline dbtTVE & \#eIDBT & 309.000 & 3 \\
\hline
\end{tabular}


BBCqt. También lo es que se muestre predispuesto a participar en las discusiones que se generan a partir del contenido de estos debates. Por el contrario, Berrocal-Gonzalo et al. afirman que la audiencia mediterránea prefiere formatos de infoentretenimiento y telerrealidad, alrededor de los cuales se registran altos índices de audiencia y audiencia social en España. A estos elementos culturales se suman los demográficos. El perfil de edad de la audiencia de los programas para los que se han obtenido datos indica que se trata de un público envejecido. Precisamente en las emisiones donde la audiencia menor de 45 años tiene más peso es donde encontramos una mayor audiencia social.

En cualquier caso, la poca capacidad mostrada por los servicios públicos multimedia para crear un debate público a través del metamedio Twitter tiene atenuantes económicos y políticos en el caso español. La falta de recursos destinados a los departamentos de nuevos medios y los obstáculos e incertidumbres legales son causas que apuntan autores como Brevini (2010) para esta situación. También Bonini y Sellas (2014) entienden que en un contexto de crisis de legitimidad de las corporaciones públicas en Europa, los programas no pueden dejar de lado su función de generar participación entre su audiencia. Por ello consideran fundamental la creación de estrategias de actuación destinadas a fidelizar al público en red para garantizar el cumplimiento de las misiones de servicio público online.

Hay indicios de que sobreimpresionar los hashtags y/o los tuits durante la emisión puede repercutir en una mayor actividad en la red

Para finalizar, esta investigación tiene limitaciones que cabe aclarar:

- se realizó una selección de programas y período temporal que pretendía ser de normalidad mediática. Sin embargo, durante el análisis tuvieron lugar sucesos, como el atentado de París o el avance del proceso de independencia de Catalunya, que alteraron esta normalidad;

- como ya aclaramos, no contamos con los datos de audiencia de la $B B C$ por su coste y la imposibilidad de acceder a ellos a través de otra fuente;

- en futuras investigaciones será necesario comparar nuestros resultados con una nueva muestra que incluya operadores privados. Ampliando el corpus podremos desvelar si estas corporaciones cumplen más eficientemente la misión de generar una esfera pública de debate.

\section{Notas}

1. Concepto que Lev Manovich (2008, p. 50) define como "un nuevo sistema semiótico y tecnológico (hibridado) que incluye técnicas y estéticas mediáticas previas entre sus elementos".

2. En la clasificación horaria televisiva del Reino Unido corresponde a la franja 20:00-22:30. Consúltese:

http://stakeholders.ofcom.org.uk/binaries/broadcast/ other-codes/tacode.pdf
3. Datos de audiencia extraídos para España de Kantar Media y de BARB para Reino Unido. Muestran la cuota de pantalla media en febrero y octubre de 2015 , respectivamente.

4. La primera semana de análisis el programa 30 minuts no se emitió.

5. Se incluyó el programa de 30 minuts emitido el día 8 de noviembre de 2015, al considerar que sus 498 usuarios activos se acercaban suficientemente al margen de 500 propuesto para la comparativa.

6. Consúltese el artículo publicado en el portal web $B B C$ Academy:

http://www.bbc.co.uk/academy/production/article/ $\operatorname{art20131008171356541}$

Los hashtags revelan información sobre la creación de debate de cada programa

\section{Bibliografía}

Anstead, Nick; O'Loughlin, Ben (2011). "The emerging viewertariat and $B B C$ question time: Television debate and real-time commenting online". The international journal of press and politics, v. 16, n.4, pp.440-462.

http://goo.gl/pgMYBf

http://dx.doi.org/10.1177/1940161211415519

Azurmendi, Ana; Llorens, Carles; López-Vidales, Nereida; Bas-Portero, Juan-José (2015): “La participación del público como valor añadido de servicio público para la televisión de proximidad. Estudio de caso de La noche de..., en ETB 2". Revista latina de comunicación social, n. 70, pp. 490-518. http://dx.doi.org/10.4185/RLCS-2015-1056

Berrocal-Gonzalo, Salomé; Redondo-García, Marta; Martín-Jiménez, Virginia; Campos-Domínguez, Eva (2014). “La presencia del infoentretenimiento en los canales generalistas de la TDT española". Revista latina de comunicación social, n. 69, pp. 85-103.

http://dx.doi.org/10.4185/RLCS-2014-1002

Bonini, Tiziano; Sellas, Toni (2014). "Twitter as a public service medium? A content analysis of the Twitter use made by Radio RAI and RNE". Communication and society, v. 27, n. 2, pp. 125-146.

http://www.unav.es/fcom/communication-society/es/ articulo.php?art_id=493

Brevini, Benedetta (2010). "Towards PSB 2.0? Applying the PSB ethos to online media in Europe: A comparative study of PSBs' internet policies in Spain, Italy and Britain". European journal of communication, v. 25, n. 4, pp. 348-365. http://dx.doi.org/10.1177/0267323110381004

Bruns, Axel (2008). Blogs, Wikipedia, Second Life, and beyond. New York, Peter Lang Publishing. ISBN: 9780820 488660

Buschow, Christopher; Schneider, Beate; Ueberheide, Simon (2014). "Tweeting television: Exploring communication activities on Twitter while watching TV". European journal of comunication research, v. 39, n. 2, pp. 129-149. 
http://goo.gl/hj8jYM

Campos-Freire, Francisco (2015). "Adaptación de los medios tradicionales a la innovación de los metamedios". El profesional de la información, v. 24, n. 4, pp. 441-450.

http://dx.doi.org/10.3145/epi.2015.jul.11

Chadwick, Andrew (2006). Internet politics: States, citizens and new communications technologies. Oxford: Oxford University Press. ISBN: 9780195177732

Curran, James; lyengar, Shanto; Lund, Anker-Brink; Salovaara-Moring, Inka (2009). "Media system, public knowledge and democracy: A comparative study". European journal of communication, v. 24, n. 1, pp. 5-26.

https://pcl.stanford.edu/research/2008/curran-mediasystems. $p d f$

http://dx.doi.org/10.1177/0267323108098943

Dahlgren, Peter (2009). "Television, public spheres and civic cultures". En: Wasko, Janet. A companion to television. Oxford: Blackwell Publishing, pp. 411-432. ISBN: 97814051 00946

Debrett, Mary (2009). "Riding the wave: public service television in the multi-platform era". Media, culture and society, v. 31, n. 5 , pp. $807-827$.

http://dx.doi.org/10.1177/0163443709339466

Enli, Gunn-Sara (2008). "Redefining public service broadcasting: multi-platform participation". Convergence: The international journal of research into new media technologies, v. 14, n. 1, pp. 105-120.

http://dx.doi.org/10.1177/1354856507084422

Fazeen, Mohamed; Dantu, Ram; Guturu, Parthasarathy (2011). "Identification of leaders, lurkers, associates and spammers in a social network: context-dependent and context-independent approaches". Social network analysis and mining, v. 1, n. 3, pp. 241-254.

http://dx.doi.org/10.1007/s13278-011-0017-9

Galindo-Rubio, Fernando; Fernández-Blanco, Elena; Alameda-García, David (2014). "Análisis del uso e interacción con aplicaciones second screen en TV social en España". Cuadernos de información, n. 35, pp. 159-175.

http://dx.doi.org/10.7764/cdi.35.565

García-Avilés, José-Alberto (2012). "Roles of audience participation in multiplatform television: From fans and consumers, to collaborators and activists". Participations, v. 9, n. 2, pp. 429-447.

http://www.participations.org/Volume\%209/Issue\%20 2/24\%20Garcia-Aviles.pdf

García-De-Torres, Elvira; Yezers'Ka, Lyudmyla; Rost, Alejandro; Calderín, Mabel; Edo, Concha; Rojano, Miladys; Said, Elías; Jerónimo, Pedro; Arcila, Carlos; Serrano, Ana; BadiIlo, Jorge; Corredoira, Loreto (2011). "Uso de Twitter y Facebook por los medios iberoamericanos". El profesional de la información, v. 20, n. 6, pp. 611-620.

http://recyt.fecyt.es/index.php/EPI/article/view/epi.2011. nov.02

http://dx.doi.org/10.3145/epi.2011.nov.02

Giglietto, Fabio; Selva, Donatella (2014). "Second screen and participation: A content analysis on a full season dataset of tweets". Journal of communication, n. 64, pp. 260277.

http://dx.doi.org/10.1111/jcom.12085

Greer, Douglas; Ferguson, Clark (2011). “Using Twitter for promotion and branding: A content analysis of local television Twitter sites". Journal of broadcasting and electronic media, v. 55, n. 2, pp.198-214.

http://dx.doi.org/10.1080/08838151.2011.570824

Gripsrud, Jostein (2007). "Television and the European public sphere". European journal of communication, v. 22, n. 4, pp. 479-492.

http://doi.org/10.1177/0267323107083064

Guerrero-Solé, Frederic (2015). “Community detection in political discussions on Twitter. An application of the retweet overlap network method to the Catalan Process toward independence". Social science computer review, November 23.

http://dx.doi.org/10.1177/0894439315617254

Guerrero-Solé, Frederic; Corominas-Murtra, Bernat; LópezGonzález, Hibai (2014). "Pacts with Twitter. Predicting voters' indecision and preferences for coalitions in multiparty systems". Information, communication and society, v. $17, \mathrm{n}$. 10, pp. 1280-1297.

http://dx.doi.org/10.1080/1369118X.2014.920040

Habermas, Jürgen (1989). The structural transformation of the public sphere. Massachusetts, DEKR. ISBN: 9780262 581080

http://goo.gl/bZzvH

Hallin, Daniel; Mancini, Paolo (2004). Comparing media systems. Three models of media and politics. New York: Cambridge University Press. ISBN: 9780521543088

Harrison, Jackie; Wessels, Bridgette (2005). "A new public service communication environment? Public service broadcasting values in the reconfiguring media". New media and society, v. 17, n. 6, pp. 34-53.

http://dx.doi.org/10.1177/1461444805058172

Jakubowicz, Karol (2010). “PSB 3.0: Reinventing European PSB". En: losifidis, Petros. Reinventing public service communication. London, Palgrave Macmillan, pp. 9-23. ISBN: 9780 230229679

http://goo.gl/mdiKgF

Larsen, Håkon (2010). “Legitimation strategies of public service broadcasters: the divergent rhetoric in Norway and Sweden". Media, culture and society, v. 32, n. 2, pp. 267283.

http://dx.doi.org/10.1177/0163443709355610

Manovich, Lev (2008). Software takes command. New York: Georgetown University. ISBN: 978623567453

http://softwarestudies.com/softbook/manovich_ softbook_11_20_2008.pdf

Quintas-Froufe, Natalia; González-Neira, Ana; Díaz-González, María-Jesús (2015). "La construcción de la estrategia comunicativa en Twitter de un falso documental: Operación Palace". Revista latina de comunicación social, v. 70, n. 1, pp. 28-48. 
http://dx.doi.org 10.4185/RLCS-2015-1033

Steemers, Jeanette (2002). "Public service broadcasting is not yet dead: survival strategies in the $21^{\text {st }}$ century". En: RIPE Conf Finland, pp. 123-136.

http://www.nordicom.gu.se/sites/default/files/kapitel$p d f / 81$ 123-136.pdf

Thomass, Barbara (2010). "PSB and the European public sphere". En: Iosifidis, Petros. Reinventing public service communication. European broadcasters and beyond. London, Palgrave Macmillan, pp. 63-76. ISBN: 9780230229679 $h t t p: / / g o o . g l / m d i K g F$

Van-Dijk, José; Poell, Thomas (2015). “Making public television social? Public service broadcasting and the challenges of social media". Television and new media, v. 16, n. 2, pp. 148-164.

http://doi.org/10.1177/1527476414527136

Walzer, Alejandra; Retis, Jéssica (2008). "Modelos de servicio público en Europa: análisis comparativo de TVE y $B B C$ ". Comunicar, v. 16, n. 31, pp. 715-726.

http://dx.doi.org/10.3916/c31-2008-03-072

\section{Colección EPI Scholar}

\section{Libros científicos de Información, Documentación y Comunicación}

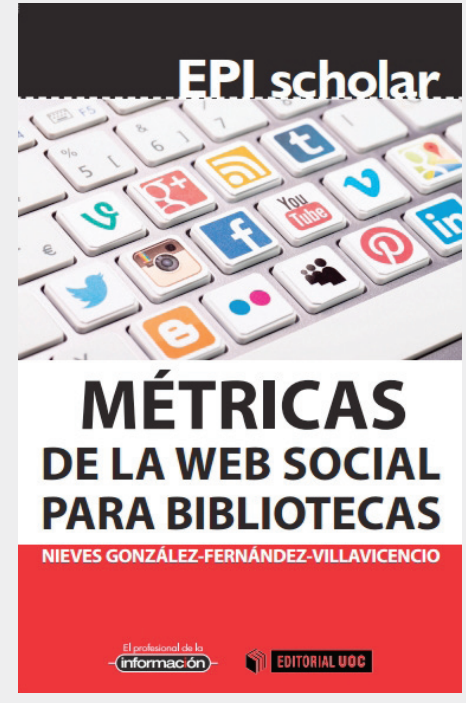

Métricas de la web social para bibliotecas de Nieves González-Fernández-Villavicencio

Métricas de la web social para bibliotecas identifica, describe y analiza los indicadores que no deben faltar en cualquier biblioteca en su plan de marketing digital y en la evaluación de su uso de los medios sociales, incluso para compararse con la competencia o entre iguales.

La rentabilidad de las acciones que llevan a cabo las bibliotecas en la web social se demuestra con métricas de impacto. Este libro señala cómo los medios sociales son buenos para conseguir beneficios ROI (económicos), pero sobre todo son muy buenos para conseguir beneficios no ROI: prestigio, reconocimiento, participación e interacción con los usuarios

González-Fernández-Villavicencio, Nieves (2015). Métricas de la web social para bibliotecas. Barcelona: El profesional de la información, Editorial UOC, colección EPI Scholar n. 4, 282 pp. ISBN: 9788491161813

\section{Calidad en sitios web. Método de análisis general, e-commerce, imágenes, hemerotecas y turismo \\ R. Pedraza-Jiménez, L. Codina y J. Guallar (coords.)}

Calidad, evaluación, análisis, sitios web... ¿Qué tienen en común todas estas palabras clave para que merezcan la atención del público de esta colección? El punto que lo une todo es la necesidad de un amplio y a la vez muy diverso colectivo profesional de saber qué es lo que hace que un sitio web responda a criterios de calidad.

Pedraza-Jiménez, Rafael; Codina, Lluís; Guallar, Javier (coords.) (2016). Calidad en sitios web. Método de análisis general, e-commerce, imágenes, hemerotecas y turismo. Barcelona: El profesional de la información, Editorial UOC, colección EPI Scholar n. 5, 184 pp. ISBN: 9788490644874

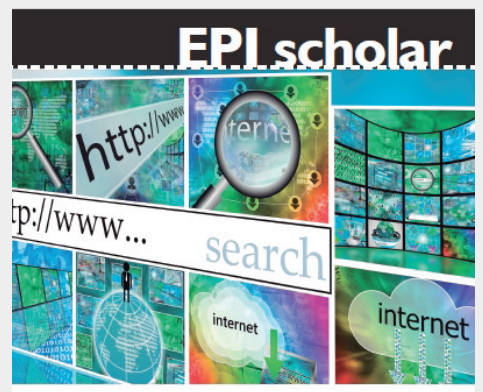

CALIDAD EN SITIOS WEB METODO DE ANALISIS GENERAL, E-COMMERCE, IMÁGENES, HEMEROTECAS Y TURISMO

RAFAEL PEDRAZA-JTMENEZ

LLUÍS CODINA

JAVIER GUALLAR (COORD.)

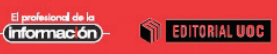

Información: Isabel Olea epi.iolea@gmail.com http://www.elprofesionaldelainformacion.com/librosEPIScholar.html 